\title{
What dreams may come?
}

\section{The scientific benefits of eating cheese before bedtime.}

\section{Paolo Mazzarello}

L ike a snake biting its tail, the circular chain of carbon atoms danced through the dreaming mind of August Kekulé. Awakened by the vision, he spent the rest of the night developing this strange idea. Shortly afterwards he came up with a simple structure for benzene, the foundation of all aromatic substances: six carbon atoms alternately linked by three single and three double bonds to form a closed chain.

This tale of discovery inspired by a dream is not the sole example of scientific creativity catalysed by sleep. Take the autobiographical sketch by Nobel prizewinner Otto Loewi. On the night before Easter Sunday 1921: "I awoke, turned on the light, and jotted down a few notes on a tiny slip of thin paper. Then I fell asleep again. It occurred to me at six o'clock in the morning that during the night I had written down something most important, but I was unable to decipher the scrawl. The next night, at three o'clock, the idea returned. It was the design of an experiment to determine whether or not the hypothesis of chemical transmission that I had uttered 17 years ago was correct. I got up immediately, went to the laboratory, and performed a simple experiment on a frog heart according to the nocturnal design."

Loewi prepared two isolated and beating frog hearts, the first with its vagus nerve, the second without. Both were filled with a Ringer saline solution. "The vagus nerve of the first heart was stimulated for a few minutes. Then the Ringer solution that had been in the first heart during the stimulation of the vagus was transferred to the second heart." This second heartbeat slowed down in the same way as it would have after a vagus stimulation.

This result, in the words of Loewi, "proved that the nerves do not influence the heart directly but liberate from their terminals specific chemical substances which, in their turn, cause the well-known modifications of the function of the heart characteristic of the stimulation of its nerve" (see Perspectives in Biology and Medicine 4, $1-25 ; 1960)$.

This was the beginning of an enormous amount of research on the chemical transmission of nerve impulses that led ultimately to a new concept of brain activity. Loewi, who was at the time professor of pharmacology at the University of Graz, where he remained until his expulsion by the Nazis in 1938, realized that, while sleeping, he had begun to link the new methods he had devel-

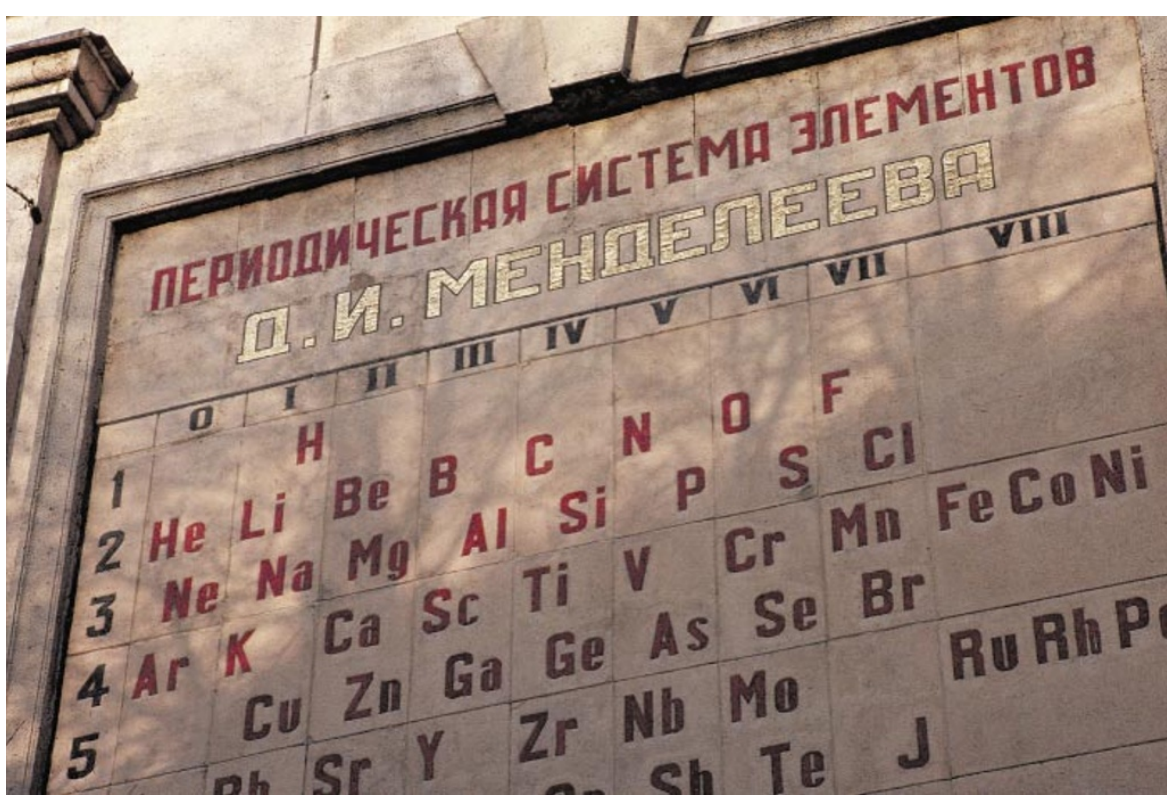

It's all coming back to me: the periodic table was revealed to Mendeleyev in a dream.

oped to study possible substances "given off from the heart" to the hitherto unproved theory of chemical transmission.

Another example of the birth of a scientific discovery during sleep is chemistry's Magna Carta, the periodic table. In February 1869, Dmitry Mendeleyev, a 35-year-old renowned professor of chemistry at the University of St Petersburg, was puzzling over a fascinating problem: could the 63 known chemical elements be ordered by an underlying rule?

The elements could be sorted into groups according to their properties, or placed in a chain according to their atomic weights. But chemists could not uncover the relationship between chemical properties and atomic weights. During an exhausting attempt to grasp the solution — also aided by cards on which he wrote the symbols and weights of the elements, a sort of game of chemical patience - Mendeleyev fell asleep. Then: "I saw in a dream a table where all the elements fell into place as required. Awakening, I immediately wrote it down on a piece of

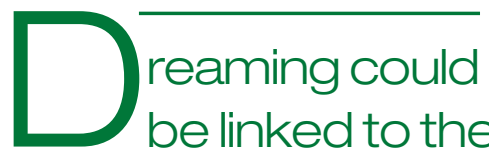

expression of new concepts only hinted at during waking. paper" (see Mendeleyev's Dream by Paul Strathern, Hamilton; 2000).

The dream had suggested an arrangement of the different groups of elements (gathered on the basis of their chemical similarities) in the order of atomic weights, allowing a regular progression with a "clear periodicity of properties" to emerge. Mendeleyev's periodic table soon became the conceptual foundation of chemical science, and immediately allowed for the prediction of hitherto unknown elements.

These three stories demonstrate that, even if rarely, scientific creativity and learning can arise during sleep. Sigmund Freud would have said that these stories are clear examples of wish-fulfilment. But a more scientific explanation is suggested by recent results obtained by Pierre Maquet et al. (Nature Neuroscience 3, 831-836; 2000). They provide evidence that memory traces are processed during REM (rapid eye movement) sleep in humans. These results seem to suggest that dreaming could be linked to a learning situation from which a new concept, only hinted at during waking experiences, could be better expressed during the dreaming reactivation and processing of memory traces, finally emerging in consciousness as a retained dream.

Paolo Mazzarello is at the Istituto di Genetica

Biochimica ed Evoluzionistica-CNR, Via Abbiategrasso 207, 27100 Pavia, Italy.

Erratum: In the Essay "Magic beans" (Nature 407, 567; 2000) the picture of boats was inverted, left to right. 